\title{
Ciclos de actividad diaria y estacional de un gremio de saurios en las dunas de arena de Viesca, Coahuila, México
}

\author{
Daily and seasonal activity patterns of a lizard guild in the sand dunes of Viesca, Coahuila, \\ Mexico
}

\author{
Cristina García-De la Peña ${ }^{1 *}$, Héctor Gadsden ${ }^{2}$, Armando J. Contreras-Balderas ${ }^{1}$ y Gamaliel Castañeda ${ }^{1}$ \\ ${ }^{1}$ Facultad de Ciencias Biológicas. Universidad Autónoma de Nuevo León. San Nicolás de los Garza, 66450, Nuevo León, México. \\ ${ }^{2}$ Instituto de Ecología, A. C., Centro Regional Chihuahua. Apartado postal 28. Carretera Chihuahua-Ojinaga, Ciudad Aldama, 32900, Chihuahua, \\ México. \\ *Correspondencia: cristina.g.delapena@gmail.com
}

\begin{abstract}
Resumen. En el 2002 se evaluaron los ciclos de actividad diaria y estacional de los saurios Uta stejnegeri, Uma exsul y Aspidoscelis marmorata en un hábitat de dunas al suroeste de Coahuila, México. Se recorrió un transecto de $500 \mathrm{~m}$, cada hora (de 0700 a 1900 h) durante 7 días, en primavera, verano y otoño. Se registró el número de individuos activos por especie, la temperatura del aire y del sustrato, y la humedad relativa. La amplitud de nicho temporal se calculó mediante el Índice estandarizado de Levins, y el traslape de nicho mediante el método de Pianka. En las 3 estaciones del año $U$. stejnegeri inició su actividad más temprano que $U$. exsul, y ésta, a su vez, se registró antes que $A$. marmorata. Las 3 especies presentaron un ciclo de actividad bimodal durante primavera y verano, y unimodal en otoño. Aspidoscelis marmorata presentó la menor amplitud de nicho temporal durante las 3 estaciones. La temperatura corporal de cada especie y la temperatura ambiental parecen determinar los patrones de actividad de este gremio de saurios en Viesca.
\end{abstract}

Palabras clave: Uta stejnegeri, Uma exsul, Aspidoscelis marmorata, nicho temporal.

\begin{abstract}
In 2002, daily and seasonal activity patterns of the lizards Uta stejnegeri, Uma exsul, and Aspidoscelis marmorata were evaluated in a dune habitat in the southwestern portion of Coahuila, Mexico. Methods included surveying a $500 \mathrm{~m}$ transect once per hour between 0700 and 1900 for 7 days each season during the spring, summer and fall. The number of active lizards, air and substrate temperatures, and relative humidity were recorded. A standardized Levins Index and Pianka's method were used to measure temporal niche breadth and overlap, respectively. In each of the 3 seasons, $U$. stejnegeri was active earlier than $U$. exsul, and $U$. exsul was active before A. marmorata. The 3 species showed a bimodal activity pattern during spring and summer, and unimodal in fall. Aspidoscelis marmorata showed the smallest niche breadth in the 3 seasons. For each species, preferred body temperature and environmental temperature seem to dictate activity patterns of this lizard guild at Viesca.
\end{abstract}

Key words: Uta stejnegeri, Uma exsul, Aspidoscelis marmorata, temporal niche.

\section{Introducción}

El estudio del reparto de los recursos disponibles en un ecosistema puede ayudar a comprender la manera en la que las especies coexisten (Pianka, 1974). El desarrollo de un período de actividad por parte de los organismos de un ecosistema es una de las estrategias que ha moldeado la estructura de las comunidades a través de la evolución;

Recibido: 13 diciembre 2005; aceptado: 06 septiembre 2006 sin embargo, los factores que desencadenan los procesos de segregación y establecen el nicho temporal de cada especie no han sido comprendidos del todo (Jaksic, 1982; Wiens et al., 1986).

La segregación temporal diaria puede favorecer la coexistencia al evitar una confrontación directa de los individuos, o bien, al reducir el traslape en el uso de los recursos (Kronfeld-Schor y Dayan, 2003). Por ejemplo, se ha observado que cuando las especies simpátricas presentan poco traslape en sus patrones de actividad diaria, la competencia por otro tipo de recursos como el alimento 
o el espacio es menor (Johnston y Zucker, 1983).

Diariamente, los saurios llevan a cabo actividades importantes como la termorregulación, la alimentación y la reproducción (Huey, 1982). El intervalo de tiempo en que los saurios están activos suele estar relacionado con el tipo de clima, la intensidad de la luz solar, la duración del fotoperíodo, la temperatura del ambiente y la hora de actividad de sus presas (House et al., 1980; Vernet et al., 1988; Díaz y Cabezas-Díaz, 2004). Asimismo, el período de actividad se relaciona directamente con la temperatura corporal de los saurios debido a que las especies que comienzan sus actividades temprano en el día y permanecen activas durante largos períodos de tiempo, generalmente presentan temperaturas corporales más bajas que aquellas especies con períodos de actividad más cortos (Pianka, 1977; Pianka et al., 1979).

En las dunas de arena de Viesca, Coahuila, habitan 8 especies de saurios, de las cuales Uta stejnegeri, Uma exsul y Aspidoscelis marmorata son las más abundantes (Castañeda et al., 2004). Cabe resaltar que $U$. exsul (lagartija de arena de Coahuila) es endémica de este lugar, su distribución es puntual y debido a la reducción de su hábitat actualmente se encuentra en la categoría de "sujeta a protección especial" de la Norma Oficial Mexicana 059 (SEMARNAT, 2001).

Para incrementar el conocimiento ecológico sobre la repartición del recurso temporal en $U$. stejnegeri, $U$. exsul y A. marmorata se planteó el objetivo de evaluar el grado de segregación de cada especie mediante la comparación de la amplitud y el traslape de nicho en primavera, verano y otoño.

\section{Materiales y métodos}

El área de estudio se ubica en la localidad de Saucillo $\left(25^{\circ} 26^{\prime} 27^{\prime}\right.$ ' N y $\left.102^{\circ} 55^{\prime} 15^{\prime} \mathrm{O}\right)$, dentro del municipio de Viesca, Coahuila. Esta región presenta una altura promedio de $1100 \mathrm{~m}$, una temperatura media anual de $21^{\circ} \mathrm{C}$ y una precipitación media anual de $250 \mathrm{~mm}$, aunque existe una fuerte variación interanual en ésta última (Gadsden et al., 2001). Las dunas de arena en esta región presentan una vegetación de tipo xerófila (Rzedowski, 1978) con dominancia de especies vegetales, como la gobernadora (Larrea tridentata), el saladillo (Suaeda nigrescens) y el mezquite (Prosopis glandulosa).

El trabajo de campo se llevó a cabo en primavera (abril), verano (agosto) y otoño (noviembre) de 2002. La toma de datos se realizó durante 7 días en cada una de las 3 estaciones. Se estableció al azar un transecto permanente de 100 por $500 \mathrm{~m}$, el cual fue recorrido cada hora (de 0700 a $1900 \mathrm{~h}$ ) por 2 personas (50 por $500 \mathrm{~m}$ cada una) durante aproximadamente 20 minutos. Durante el trayecto se registró el número de individuos por especie (sin considerar clase de edad ni sexo) que se encontraron activos. Cada una de las 3 especies fue fácilmente distinguible por su morfología y coloración. A cada hora se registró la humedad relativa y la temperatura del aire a $15 \mathrm{~cm}$ de la superficie (usando un termohigrómetro Exthech $®$ ), y la temperatura del sustrato en sol directo (mediante un termómetro láser para superficies Raynger®). La amplitud de nicho temporal se calculó para cada día mediante el índice de Levins (1968) estandarizado por Hurlbert (1978) cuyas ecuaciones son:

$$
\begin{gathered}
B=\frac{1}{\sum p_{j}^{2}} \\
p_{j}=\frac{N_{i}-}{Y} \\
B s=\frac{B-1}{n-1}
\end{gathered}
$$

donde $B=$ índice de Levins, $p_{j}=$ proporción de individuos de una especie en un período de tiempo $j, N_{j}=$ número de individuos de una especie en un período de tiempo $j$, $Y=$ número total de individuos en la muestra, $B S=$ índice estandarizado de Levins (escala de $0=$ mínima amplitud de nicho a $1=$ máxima amplitud de nicho) y $n=$ número total de recursos (horas).

El traslape de nicho se calculó para cada día mediante el método de Pianka (1973), cuya ecuación es:

$$
O_{j k}=\frac{\sum p_{i j}}{\sqrt{\sum p_{i j}^{2}} \sum \underline{p}^{\frac{p_{k}}{p_{i k}}}{ }^{2}}
$$

donde $O_{j k}=$ índice de traslape de nicho de Pianka entre las especies $j$ y $k, p_{i j}=$ proporción que representa el intervalo de tiempo $i$ del total de intervalos utilizados por la especie $j, p_{i k}=$ proporción que representa el intervalo de tiempo $i$ del total de intervalos utilizados por la especie $k$ y $n=$ número total de recursos (horas).

Se utilizaron pruebas de Kolmogorov-Smirnov para comprobar la normalidad de los datos ambientales y de la amplitud y traslape de nicho obtenidos durante los 7 días de cada estación. Debido a que los datos resultaron normales se aplicaron Análisis de Varianza (ANOVA) de un factor para encontrar diferencias en las variables ambientales entre estaciones, así como en la amplitud y el traslape de nicho entre especies para cada estación y entre estaciones para cada especie. Finalmente se utilizaron pruebas de comparaciones múltiples (Tukey) cuando fue necesario. Las medias se muestran como $\bar{x} \pm$ error estándar. Todas las pruebas se consideraron significativas con $\mathrm{P}<0.05$. Los 
modelos ecológicos fueron calculados con el programa Ecological Methodology@ Ver. 5.2 (Krebs, 1999, 2000).

\section{Resultados}

\section{Comparaciones Intraestacionales}

Primavera. Se observó un patrón de actividad bimodal para las 3 especies de saurios (Fig. 1). Uta stejnegeri y Uma exsul iniciaron su actividad a las $0800 \mathrm{~h}$ en primavera, y Aspidoscelis marmorata inició su actividad a las 0900 h. El primer y más importante intervalo de alta actividad para U. stejnegeri y $U$. exsul se registró entre las 0900 y $1100 \mathrm{~h}$, mientras que para $A$. marmorata fue de 1000 a $1300 \mathrm{~h}$. Entre las 1400 y $1500 \mathrm{~h}$ ambas temperaturas del medio fueron altas (aire: $\bar{x}=34.6 \pm 0.5^{\circ} \mathrm{C}$, sustrato: $\bar{x}=$ $\left.52.1 \pm 1.4^{\circ} \mathrm{C}\right)$ y la humedad relativa presentó valores bajos $(\bar{x}=11.4 \pm 1.3 \%)$, todas ellas en comparación con las horas anteriores. En estas 2 horas las proporciones de individuos activos de $U$. stejnegeri y $U$. exsul fueron las más bajas del día. El segundo y menor intervalo de alta actividad para las 3 especies se observó entre las 1600 y 1800 h. Las amplitudes del nicho temporal calculadas para $U$. stejnegeri y $U$. exsul fueron significativamente más altas que la amplitud obtenida para $A$. marmorata (Cuadro 1), $\mathrm{F}_{2,18}=24.5, \mathrm{P}=0.0001$. Sin embargo, no se observó
Cuadro 1. Media de la amplitud de nicho temporal de Uta stejnegeri, Uma exsul y Aspidoscelis marmorata en tres estaciones del año. Media \pm Error estándar.

\begin{tabular}{lccc}
\hline Estación & U. stejnegeri & U. exsul & A. marmorata \\
\hline Primavera & $0.56 \pm 0.1$ & $0.50 \pm 0.3$ & $0.34 \pm 0.1$ \\
Verano & $0.70 \pm 0.3$ & $0.69 \pm 0.1$ & $0.52 \pm 0.2$ \\
Otoño & $0.55 \pm 0.1$ & $0.50 \pm 0.2$ & $0.38 \pm 0.1$ \\
\hline
\end{tabular}

Cuadro 2. Media del traslape de nicho temporal de Uta stejnegeri, Uma exsul y Aspidoscelis marmorata en tres estaciones del año. Media \pm error estándar.

\begin{tabular}{llll}
\hline Estación & $\begin{array}{l}\text { U. stejnegeri- } \\
\text { U. exsul }\end{array}$ & $\begin{array}{l}\text { U. stejnegeri- } \\
\text { A. marmorata }\end{array}$ & $\begin{array}{l}\text { U. exsul- } A \text { marmorata } \\
\text { marmera }\end{array}$ \\
\hline Primavera & $0.88 \pm 0.2$ & $0.73 \pm 0.5$ & $0.75 \pm 0.4$ \\
Verano & $0.84 \pm 0.2$ & $0.76 \pm 0.2$ & $0.79 \pm 0.3$ \\
Otoño & $0.84 \pm 0.1$ & $0.81 \pm 0.007$ & $0.82 \pm 0.009$ \\
\hline
\end{tabular}

diferencia significativa entre los traslapes de nicho para las 3 posibles combinaciones de especies (Cuadro 2), $\mathrm{F}_{2,18}=$ $3.42, \mathrm{P}=0.06$.

Verano. Las 3 especies mostraron un patrón de actividad

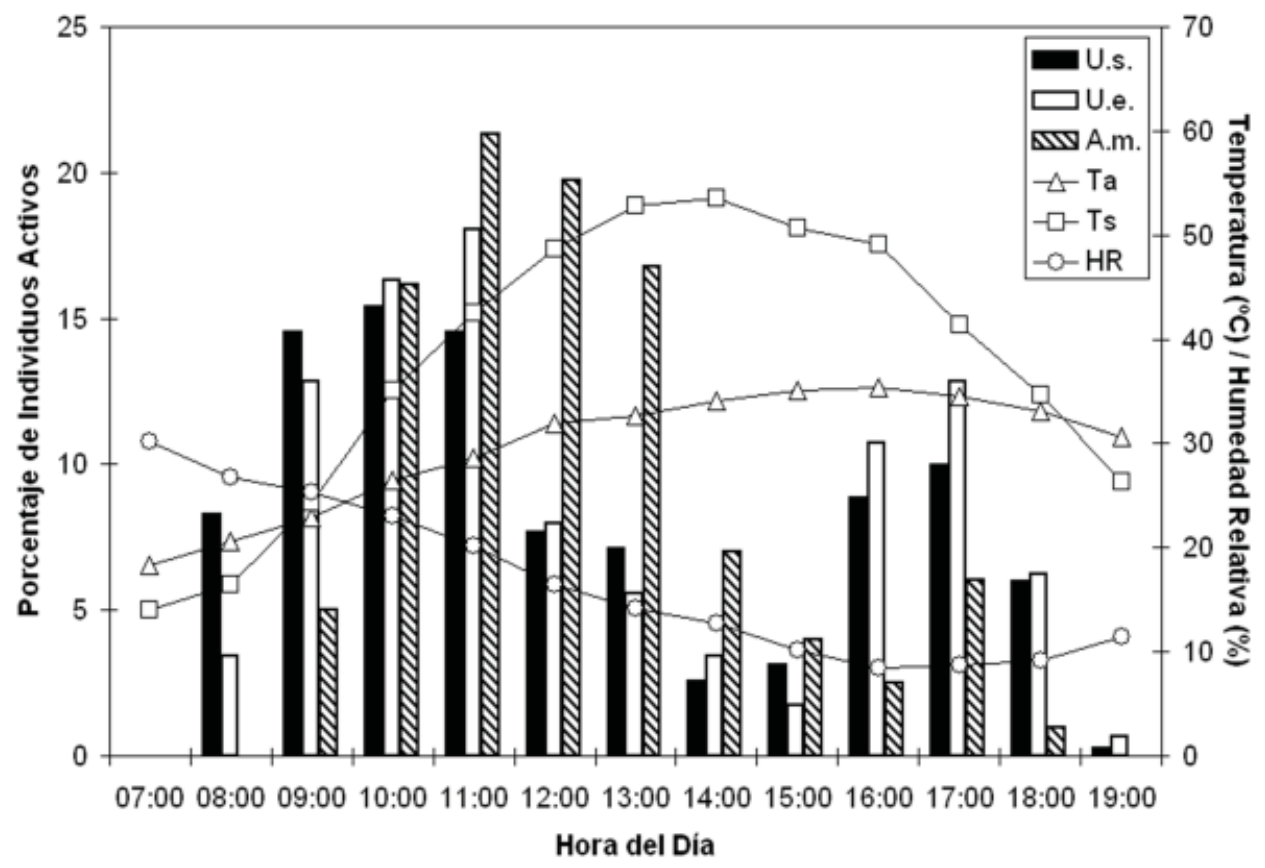

Figura 1. Porcentaje promedio de individuos de Uta stejnegeri (U.s.), Uma exsul (U.e.) y Aspidoscelis marmorata (A.m.) activos a cada hora del día en primavera. $\mathrm{Ta}=$ Temperatura del aire, $\mathrm{Ts}=$ Temperatura del sustrato, $\mathrm{HR}=$ Humedad relativa. 
bimodal (Fig. 2). De las 0700 a las $1000 \mathrm{~h}$ las temperaturas del aire y del sustrato fueron más altas en el verano (aire $\bar{x}=27.1 \pm 0.5^{\circ} \mathrm{C}$, sustrato $\bar{x}=27.4 \pm 1.0^{\circ} \mathrm{C}$ ) en comparación con las de primavera (aire $\bar{x}=22.0 \pm 0.5^{\circ} \mathrm{C}$, sustrato $\bar{x}=$ $22.5 \pm 1.5^{\circ} \mathrm{C}$ ), (aire: $\mathrm{F}_{1.54}=38.57, \mathrm{P}=0.0001$; sustrato: $\left.\mathrm{F}_{1,54}=6.97, \mathrm{P}=0.01\right)$, y la actividad de las 3 especies comenzó una hora más temprano (U. stejnegeri y U. exsul a las 0700 h y $A$. marmorata a las 0800 h). El primero y más importante intervalo de alta actividad fue más amplio que el de la primavera para las 3 especies. Las mayores proporciones de $U$. stejnegeri y $U$. exsul se registraron entre las 0800 y $1200 \mathrm{~h}$, mientras que la mayoría de los individuos de $A$. marmorata se observaron de 0900 a 1300 h. A las $1400 \mathrm{~h}$ se registraron los valores máximos de temperatura del aire $\left(\bar{x}=38.1 \pm 0.7^{\circ} \mathrm{C}\right)$ y del sustrato $(\bar{x}=$ $52.1 \pm 1.3^{\circ} \mathrm{C}$ ) que coincidieron con la menor proporción de individuos observados durante el día en las 3 especies. La actividad volvió a incrementarse a partir de las 1500 h. De 1600 a 1800 h se observó el segundo intervalo de alta actividad para U. stejnegeri y U. exsul. Sin embargo, la proporción de individuos de A. marmorata descendió conforme avanzaron las horas de la tarde. Las amplitudes de nicho calculadas para U. stejnegeri y U. exsul fueron significativamente más altas que la amplitud obtenida para A. marmorata (Cuadro 1), $\mathrm{F}_{2,18}=17.36, \mathrm{P}=0.0001$. No obstante, no se observó diferencia significativa entre los traslapes de nicho para los 3 posibles pares de especies (Cuadro 2), $\mathrm{F}_{2,18}=2.17, \mathrm{P}=0.14$.

Otoño. En esta estación se observó un patrón de actividad unimodal para las 3 especies de saurios (Fig. 3). Las temperaturas del aire y del sustrato (de las 0700 a las 1900 h) fueron más bajas en esta estación en comparación con las de primavera y verano [aire (primavera: $\bar{x}=29.5 \pm 0.5^{\circ}$ $\mathrm{C}$, verano: $\bar{x}=33.1 \pm 0.4^{\circ} \mathrm{C}$, otoño: $\bar{x}=25.9 \pm 0.6^{\circ} \mathrm{C}$; $\mathrm{F}_{2,270}=37.58, \mathrm{P}=0.0001$ ), sustrato (primavera: $\bar{x}=37.6 \pm$ $1.3^{\circ} \mathrm{C}$, verano: $\bar{x}=39.0 \pm 1.0^{\circ} \mathrm{C}$, otoño: $\bar{x}=28.5 \pm 1.2^{\circ} \mathrm{C}$; $\left.\left.\mathrm{F}_{2,270}=21.62, \mathrm{P}=0.0001\right)\right]$. La actividad de $U$. stejnegeri comenzó a las $0800 \mathrm{~h}$, la de $U$. exsul a las $0900 \mathrm{~h}$ y la de $A$. marmorata a las 1000 h. El intervalo de mayor actividad para las 3 especies fue de las 1100 a las 1500 h, durante las cuales se registraron los valores más altos de temperatura del aire y del sustrato. La actividad cesó a las 1700 h para A. marmorata y a las $1800 \mathrm{~h}$ para U. stejnegeri y U. exsul. Las amplitudes de nicho calculadas para $U$. stejnegeri y $U$. exsul fueron significativamente más altas que la amplitud obtenida para A. marmorata (Cuadro 1), $\mathrm{F}_{2,18}=23.97, \mathrm{P}=$ 0.0001 . No se observó diferencia significativa entre los traslapes de nicho para los 3 posibles pares de especies (Cuadro 2), $\mathrm{F}_{2,18}=2.28, \mathrm{P}=0.13$.

\section{Comparaciones Interestacionales}

La amplitud de nicho fue significativamente más alta en verano que en primavera y otoño para cada especie de saurio ( $U$. exsul: $\mathrm{F}_{2,18}=23.38, \mathrm{P}=0.0001 ;$ U. stejnegeri: $\mathrm{F}_{2,18}=14.3, \mathrm{P}=0.0001 ;$ A. marmorata: $\mathrm{F}_{2,18}=19.92, \mathrm{P}=$ 0.0001). No se observó diferencia significativa en los valores de traslape de nicho entre las 3 estaciones para cada combinación de especies (U. stejnegeri $-U$. exsul: $\mathrm{F}_{2,18}=1.00, \mathrm{P}=0.38 ;$ U. stejnegeri - A. marmorata: $\mathrm{F}_{2,18}=$ $1.43, \mathrm{P}=0.26 ;$ U. exsul - A. marmorata: $\mathrm{F}_{2,18}=1.19, \mathrm{P}=$ $0.32)$.

\section{Discusión}

Uta stejnegeri fue la primera especie que emergió en el día, y según Parker y Pianka (1975), la media de la temperatura corporal de una especie muy cercana a ella (Uta stansburiana) es de $35^{\circ} \mathrm{C}$. Uma exsul, cuya temperatura corporal media también es de $35^{\circ} \mathrm{C}$, según García-De la Peña et al. (2005), fue la segunda en aparecer. La última fue $A$. marmorata; los registros de su temperatura corporal media son superiores a 39.7 C (Pianka, 1970; Maury, 1981). De acuerdo con Pianka (1969, 1973, 1977), el aumento paulatino en las temperaturas del aire y del sustrato que se presenta conforme avanzan las horas del día determina el orden de aparición de las especies de saurios. De esta manera, las especies que emergen más temprano tienden a presentar menores temperaturas corporales que las que aparecen más tarde. Este patrón general de relación se observó en el gremio estudiado durante las 3 estaciones. Por otra parte, la hora de emergencia de todas las especies de saurios en las estaciones cálidas se recorre cierto tiempo en las estaciones frías, permitiendo que la temperatura ambiental aumente (Pianka, 1969; Heatwole, 1970; Pianka, 1970). En este estudio la hora de emergencia y la proporción de individuos activos de cada especie se modificaron según la estación, lo que parece estar relacionado con las temperaturas del aire y del suelo, ya que en verano (estación que presentó las más altas temperaturas) las 3 especies comenzaron su actividad entre las 0700 y 0800 h, mientras que en otoño (estación más fría) el inicio de su actividad se registró entre las 0800 y las 1000 h.

Las especies que presentan un patrón de actividad bimodal durante los meses cálidos (intervalos de alta actividad en la mañana y en la tarde) con frecuencia cambian su actividad a un patrón unimodal (un solo intervalo de actividad) durante los meses fríos (Tinkle, 1967; Pianka y Parker, 1972; Pianka, 1993). Según Pianka (1993) estos cambios estacionales en el tiempo de actividad facilitan la termorregulación, ya que los saurios pueden encontrar un ambiente térmico más propicio para regular su temperatura de manera eficiente en los diferentes períodos del año. Los patrones de actividad diaria del 


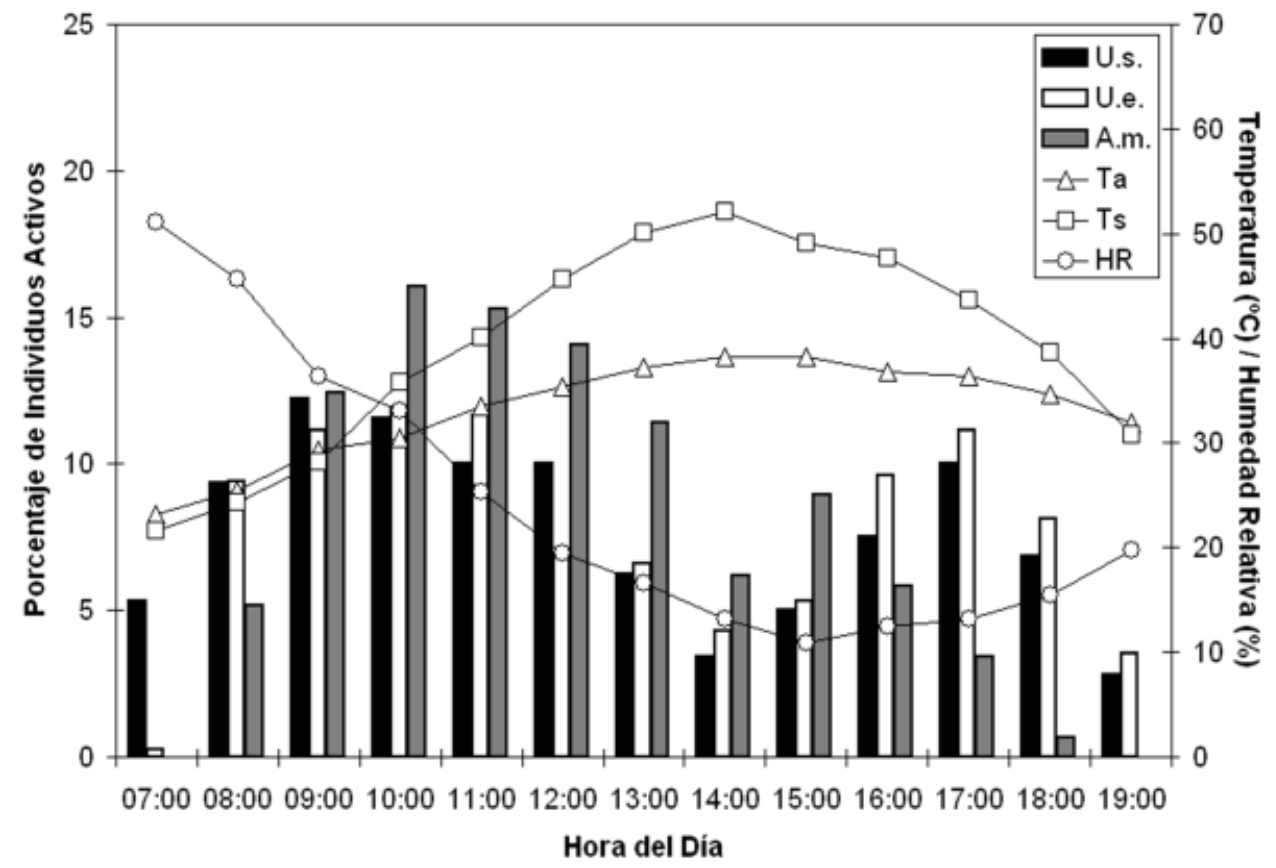

Figura 2. Porcentaje promedio de individuos de Uta stejnegeri (U.s.), Uma exsul (U.e.) y Aspidoscelis marmorata (A.m.) activos a cada hora del día en verano. Ta $=$ Temperatura del aire, $\mathrm{Ts}=$ Temperatura del sustrato, $\mathrm{HR}=$ Humedad relativa.

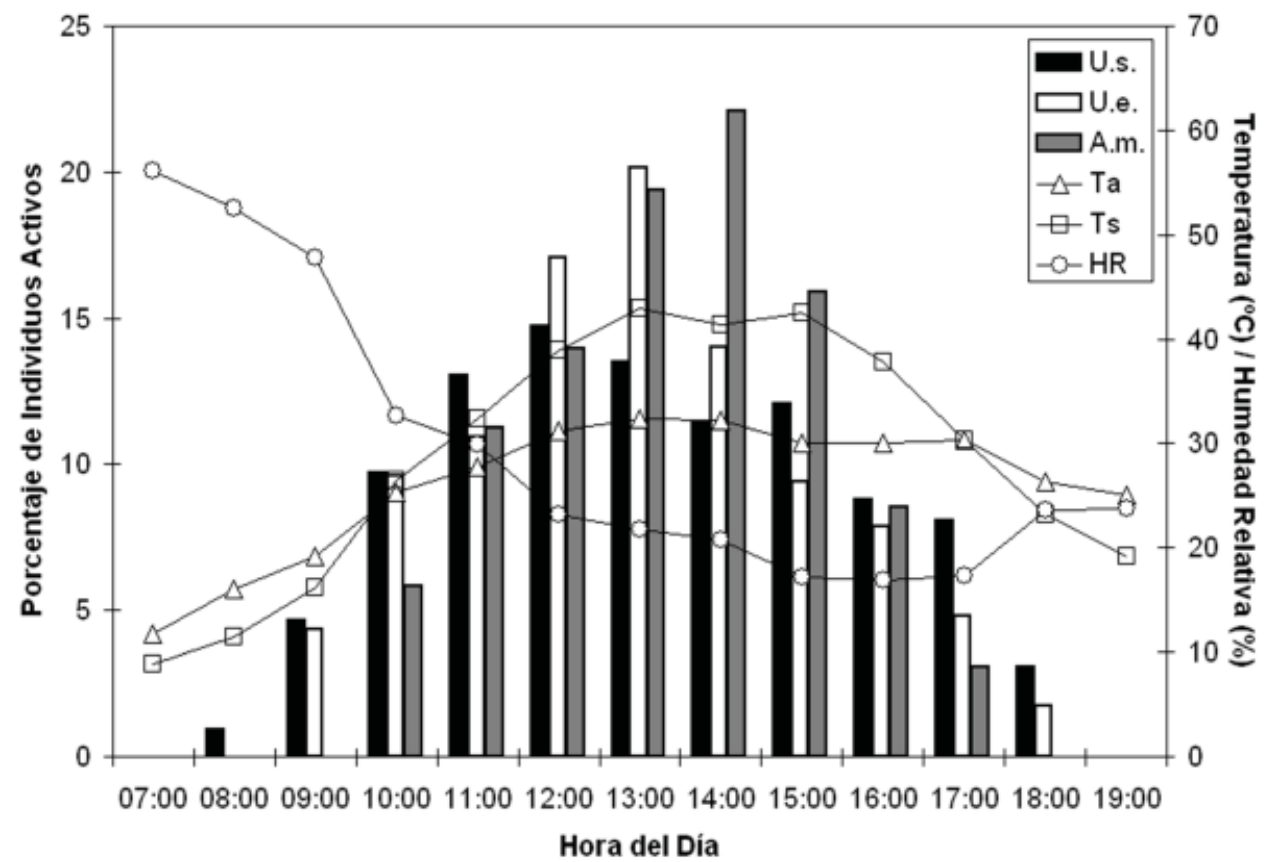

Figura 3. Porcentaje promedio de individuos de Uta stejnegeri (U.s.), Uma exsul (U.e.) y Aspidoscelis marmorata (A.m.) activos a cada hora del día en otoño. Ta $=$ Temperatura del aire, $\mathrm{Ts}=$ Temperatura del sustrato, HR $=$ Humedad relativa. 
gremio de lagartijas de Viesca cambiaron de acuerdo con la estación del año. En primavera y verano la actividad de las 3 especies estudiadas fue bimodal. La mayoría de los individuos se refugiaron durante las horas más cálidas del mediodía, posiblemente para evitar un estrés térmico e hídrico (Porter et al., 1973; Bowker, 1993). Esta conducta ha sido observada en U. stansburiana (Parker y Pianka, 1975) y en otras especies de saurios, como Sceloporus grammicus, S. scalaris (Ortega et al., 1984), Uma notata, Callisaurus draconoides y Dipsosaurus dorsalis (Ortega et al., 1986). En contraste, durante la estación de otoño, las 3 especies recurrieron a una actividad unimodal debido a que las temperaturas ambientales registradas a lo largo del día fueron más bajas que en primavera y verano, y por consecuencia se encontró una mayor cantidad de saurios de las 3 especies en las horas más cálidas del mediodía.

Los valores de amplitud de nicho temporal para las 3 especies fueron mayores en el verano en comparación con los calculados para primavera y otoño. Las altas temperaturas ambientales de esta estación parecen favorecer la rápida adquisición y mantenimiento de las temperaturas corporales óptimas para cada especie que conforma el gremio y se refleja en amplios períodos de actividad.

En las 3 estaciones se observó una mayor proporción de individuos activos de $A$. marmorata entre las $1100 \mathrm{y}$ las $1300 \mathrm{~h}$, y su amplitud de nicho fue significativamente menor que en $U$. stejnegeri y $U$. exsul. Esta diferencia en el período de actividad de $A$. marmorata puede deberse a que es un forrajero activo que requiere de una temperatura corporal elevada para llevar a cabo sus actividades de manera eficiente a diferencia de las especies de forrajeo pasivo (Bowker et al., 1986; Vitt y Morato-De Carvalho, 1995), como es el caso de las otras 2 especies estudiadas. De esta forma, las altas temperaturas del aire y del suelo que se registraron al mediodía, favorecen el mantenimiento de una alta temperatura corporal en $A$. marmorata, lo que a su vez promueve su actividad. En contraste, $U$. stejnegeri y $U$. exsul presentan temperaturas corporales más bajas que $A$. marmorata y no requieren temperaturas ambientales muy elevadas para alcanzar y mantener su propia temperatura. Esto les permite mantenerse activas durante períodos más largos en el día.

En conclusión, la temperatura corporal propia de cada especie y la temperatura del medio ambiente, son los factores que parecen determinar los patrones de actividad diaria y estacional del gremio de saurios estudiado en Viesca.

\section{Agradecimientos}

Agradecemos al Consejo Nacional de Ciencia y Tecnología (CONACYT) el apoyo financiero (beca de postgrado) a C.G.P.y G.C. A The Southwestern Association of Naturalists por otorgar el Howard McCarley Student Research Award 2004 a C.G.P. y con el cual se financió parte de este proyecto. A A. Jaime-Rosales, I. RiveraSáenz, A. Ríos-Saldaña, G. Mata-Flores y A. SánchezAlmazán por su cooperación en el trabajo de campo. A Cameron W. Barrows por su ayuda en la traducción del resumen y a dos revisores anónimos, quienes con sus sugerencias mejoraron este escrito.

\section{Literatura citada}

Bowker, R. G. 1993. The thermoregulation of the lizards Cnemidophorus exsanguis and $C$. velox: some consequences of high body temperature. In Biology of the whiptail lizards, J. W. Wright and L. J. Vitt (eds.). Oklahoma Museum of Natural History, Norman, p. 117-132.

Bowker, R. G., S. Damschroder, A. M. Sweet y D. K. Anderson. 1986. Thermoregulatory behavior of the North American lizards Cnemidophorus velox and Sceloporus undulatus. Amphibia-Reptilia 7: 335-346.

Castañeda, G., C. García-De la Peña y D. Lazcano. 2004. Notes on Herpetofauna 5: Herpetofauna of the sand dunes of Viesca, Coahuila, Mexico. Bulletin of the Chicago Herpetological Society 39: 65-68.

Díaz, J. A. y S. Cabezas-Díaz. 2004. Seasonal variation in the contribution of different behavioural mechanisms to lizard thermoregulation. Functional Ecology 18: 867-875.

Gadsden, H., H. López-Corrujedo, J. L. Estrada-Rodríguez y U. Romero-Méndez. 2001. Biología poblacional de la lagartija de arena de Coahuila Uma exsul (Sauria: Phrynosomatidae): implicaciones para su conservación. Boletín de la Sociedad Herpetológica Mexicana 9: 5166.

García-De la Peña, C., H. Gadsden, H. López-Corrujedo y D. Lazcano. 2005. Uma exsul (Coahuila Fringetoed Sand Lizard). Body Temperature. Herpetological Review 36: 66-67.

Heatwole, H. 1970. Thermal ecology of the desert dragon, Amphibolurus inermis. Ecological Monographs 40: 425-457.

House, S. M., P. J. Taylor y I. F. Spellerberg. 1980. Patterns of daily behavior in two lizards species Lacerta agilis L. and Lacerta vivipara Jacquin. Oecologia 44: 396402.

Huey, R. B. 1982. Temperature, physiology, and the ecology of reptiles. In Biology of the Reptilia, vol. 12, C. Gans and F. H. Pough (eds.). Academic, London and New York. p. 25-91. 
Hurlbert, S. H. 1978. The measurement of niche overlap and some relatives. Ecology 59: 67-77.

Jaksic, F. M. 1982. Inadequacy of activity time as a niche difference: the case of diurnal and nocturnal raptors. Oecologia 52:171-75.

Johnston, P. G. y I. Zucker. 1983. Lability and diversity of circadian rhythms of the cotton rat Sigmodon hispidus. American Journal of Physiology-Regulatory Integrative and Comparative Physiology 244: R338-46.

Krebs, C. J. 1999. Ecological methodology, segunda edición. Benjamin Cummings, Menlo Park, California. $620 \mathrm{p}$.

Krebs, C. J. 2000. Programs for ecological methodology. Ver. 5.2. (Multi-Media CD-Rom).

Kronfeld-Schor, N. y T. Dayan. 2003. Partitioning of time as an ecological resource. Annual Review of Ecology, Evolution and Systematics 34: 153-181.

Levins, R. 1968. Evolution in changing environments: some theoretical explorations. Princeton University, New Jersey. ix +120 p.

Maury, M. E. 1981. Variability of activity cycles in some species of lizards in the Bolsón de Mapimí (Chihuahuan Desert, Mexico). In Ecology of the Chihuahuan Desert: Organization of some vertebrate communities, R. Barbault and G. Halffter (eds.). Publicaciones del Instituto de Ecología, A. C. 8. México, D. F. p 101118.

Ortega, A., R. Rodríguez, L. Hernández y R. Barbault. 1984. Cycles journaliers d'activité chez deux espèces sympatriques de Sceloporus (Iguanidae), S. grammicus et $S$. scalaris. Amphibia-Reptilia 5: 347-354.

Ortega, A., A. González-Romero y R. Barbault. 1986. Rythmes journaliers d'activité et partage des ressources dans une communauté de lézards du désert de Sonora, Mexique. Revue Ecologie Terre Vie 41: 355-360.

Parker, W. S. y E. R. Pianka. 1975. Comparative ecology of populations of the lizard Uta stansburiana. Copeia 1975: 615-632.

Pianka, E. R. 1969. Sympatry of desert lizards (Ctenotus) in western Australia. Ecology 50: 1012-1030.

Pianka, E. R. 1970. Comparative autoecology of the lizard Cnemidophorus tigris in different parts of its geographic range. Ecology 51: 703-720.

Pianka, E. R. 1973. The structure of lizard communities. Annual Review of Ecology and Systematics. 4: 53-74.

Pianka, E. R. 1974. Niche overlap and diffuse competition. Proceedings of the National Academy of Science USA. 17: 2141-2145.
Pianka, E. R. 1977. Reptilian species diversity. In Biology of the Reptilia, C. Gans and D. W. Tinkle (eds.). Academic, New York. p. 1-34.

Pianka, E. R. 1993. The many dimensions of a lizard's ecological niche. In Lacertids of the Mediterranean region: a biological approach, E. D. Valakos, W. Bohme, V. Pérez-Mellado and P. Maragou (eds.). Hellenic Zoological Society, Kifissia, Attica. p. 121154.

Pianka, E. R., R. B. Huey y L. R. Lawlor. 1979. Niche segregation in desert lizards. In Analysis of Ecological Systems, D. J. Horn, R. Mitchell and G. R. Stairs (eds.). Ohio State University, Columbus, p. 67-115.

Pianka, E. R. y W. S. Parker. 1972. Ecology of the iguanid lizard Callisaurus draconoides. Copeia 1972: 493508.

Porter, W. P., J. W. Mitchell, W. A. Beckman y C. B. DeWitt. 1973. Behavioral implications of mechanistic ecology: thermal and behavioral modeling of desert ectotherms and their microenvironment. Oecologia 13:1-54.

Rzedowski, J. 1978. Vegetación de México. Limusa. México, D.F. 432 p.

SEMARNAT (Secretaría de Medio Ambiente y Recursos Naturales). 2001. Norma Oficial Mexicana (NOMECOL-059-2001). Protección ambiental-Especies nativas de México de flora y fauna silvestres-Categorías de riesgo y especificaciones para su inclusión, exclusión o cambio-Lista de especies en riesgo. Diario Oficial de la Federación (6 de marzo del 2002), México, D. F. México.

Tinkle, D. W. 1967. The life and demography of the sideblotched lizard, Uta stansburiana. Miscellaneous Publications Museum of Zoology University of Michigan (132):1-182.

Vernet, R., M. Lemire, C. Grenot, y J. Francaz. 1988. Ecophysiological comparisons between two large Saharan lizard, Uromastix acanthinurus (Agamidae) and Varanus griseus (Varanidae). Journal of Arid Environments 14: 187-200.

Vitt, L. J. y C. Morato-De Carvalho. 1995. Niche partitioning in a tropical wet season: Lizards in the Lavrado area of northern Brazil. Copeia 1995: 305-329.

Wiens. J. A., J. F. Addicot, T. J. Case y J. Diamond. 1986. Overview: the importance of spatial and temporal scale in ecological investigation. In Community ecology, J. Diamond, and T. J. Case (eds.). Harper and Row, New York. p. 45-53. 